\title{
Dynamics and Patterns of a Diffusive Prey-Predator System with a Group Defense for Prey
}

\author{
Honglan Zhu ${ }^{1}{ }^{1}$ and Xuebing Zhang $\mathbb{D}^{2}$ \\ ${ }^{1}$ Business School, Huaiyin Institute of Technology, Huaian, China \\ ${ }^{2}$ College of Mathematics and Statistics, Nanjing University of Information Science and Technology, Nanjing, China \\ Correspondence should be addressed to Xuebing Zhang; zxb1030@163.com
}

Received 11 September 2017; Accepted 26 November 2017; Published 8 January 2018

Academic Editor: Chris Goodrich

Copyright ( 2018 Honglan Zhu and Xuebing Zhang. This is an open access article distributed under the Creative Commons Attribution License, which permits unrestricted use, distribution, and reproduction in any medium, provided the original work is properly cited.

\begin{abstract}
We study a diffusive prey-predator system with a group defense for prey. Under Neumann boundary condition, we analyze local and stability of nonnegative constant steady states and the existence and nonexistence of nonconstant steady states. These results also exhibit the critical role of the system parameters leading to the formation of spatiotemporal patterns.
\end{abstract}

\section{Introduction}

The predator-prey system first proposed by $[1,2]$ is one of the fundamental ecological systems in both ecology and mathematical ecology. Based on different settings, various types of predator-prey models described by differential systems have been proposed and the dynamics of these systems are studied [3-6]. The basic form of these models is as follows:

$$
\begin{aligned}
& \frac{d x}{d t}=r x\left(1-\frac{x}{K}\right)-P(x, y), \\
& \frac{d y}{d t}=-s y+c P(x, y),
\end{aligned}
$$

where $r$ is the intrinsic growth rate and $K$ is the environmental carrying capacity of prey population, and the function $P(x)$ is the functional response; the constant $c(>0)$ is the ratio of biomass conversion and $s$ is the natural death rate of predator species. The simplest functional response is LotkaVolterra function which is described as

$$
P(x, y)= \begin{cases}a x y, & 0 \leq x \leq \frac{k}{a}, \\ k y, & x \geq \frac{k}{a},\end{cases}
$$

which is also called Holling type I function. However, the curve defined by the Lotka-Volterra response function is a straight line through the origin and is unbounded. Thus, more reasonable response functions should be nonlinear and bounded. In 1913, Michaelis and Menten proposed the response function

$$
P(x, y)=\frac{m x y}{a+x},
$$

where $m>0$ denotes the maximal growth rate of the species and $a>0$ is the half-saturation constant. It is now referred to as a Michaelis-Menten function or a Holling type II function. Another class of response function is

$$
P(x, y)=\frac{m x^{2} y}{a+b x+x^{2}}
$$

which is called a sigmoidal response function, while the simplification

$$
P(x, y)=\frac{m x^{2} y}{a+x^{2}}
$$

is known as a Holling type III function. Some authors $[7,8]$ considered system (1) with following response function:

$$
P(x, y)=\frac{m x y}{a+x^{2}}
$$


which is called Holling type IV function. Besides, Beddington-DeAngelis type $P(x, y)=\beta x y /(\alpha+x+m y)$ and more complicated functional response $P(x, y)=\beta x^{2} y /\left(x^{2}+m y^{2}\right)$ are also considered by some researchers $[9,10]$.

Recently, some works consider the case when animals join together in herds in order to provide a self-defense from predators. In [11], the authors argued that it is more appropriate to model the response functions of prey that exhibit herd behavior in terms of the square root of the prey population. Inspired by this thought, the authors in [12] choose response function $P(x)=\sqrt{x}$ to reflect this fact. When motion is allowed, [13] considered the spatiotemporal behavior of a prey-predator system with a group defense for prey by means of extensive computer simulations. The proposed model is as follows:

$$
\begin{aligned}
\frac{\partial u}{\partial t} & =d_{1} \Delta u+r_{1} u\left(1-\frac{u}{K}\right)-\rho u^{\alpha} v, \\
\quad(x, t) \in \Omega \times(0,+\infty), & \\
\frac{\partial v}{\partial t} & =d_{2} \Delta v+\beta u^{\alpha} v-r_{2} v, \quad(x, t) \in \Omega \times(0,+\infty) \\
u(x, 0) & =u_{0}(x), \\
v(x, 0) & =v_{0}(x),
\end{aligned}
$$

$$
x \in \Omega,
$$

where $u$ and $v$ denote, respectively, the densities of prey and predator species. $r$ is the growth rate of prey species, $K$ is its carrying capacity, $r_{2}$ is the mortality rate of predator species, $\rho$ is the search efficiency of predator for prey, $\beta$ is the biomass conversion coefficient, and $\alpha \in(0,1)$ represents a kind of aggregation efficiency. The local dynamics for nonspatial model was studied, such as Hopf bifurcation and existence of extinction domain. For model (7), the authors only give some numerical simulations to find some spatiotemporal features. Reference [14] considers the direction and the stability of the bifurcating periodic solutions for model (7) with $\alpha=1 / 2$ under Neumann boundary conditions. Reference [15] investigated the global dynamics of nonspatial model including the nonexistence of periodic orbits and the existence and uniqueness of limit cycles. We refer readers to [16-21] as some other related works on predator-prey model with herd behavior.

It is noted that up to now no one has studied the existence and nonexistence of positive steady state solutions of (7). Therefore, the main aim of this article is to study the existence and nonexistence of nonconstant positive solutions of the following elliptic system:

$$
\begin{aligned}
-d_{1} \Delta u & =r_{1} u\left(1-\frac{u}{K}\right)-\rho u^{\alpha} v, \quad \text { in } \Omega, \\
-d_{2} \Delta v & =\beta u^{\alpha} v-r_{2} v, \quad \text { in } \Omega, \\
\frac{\partial u}{\partial v} & =\frac{\partial v}{\partial v}=0, \quad \text { on } \partial \Omega,
\end{aligned}
$$

where $v$ is the outward unit normal vector on $\partial \Omega$, and we impose a homogeneous Neumann type boundary condition, which implies that (8) is a closed system and has no flux across the boundary $\partial \Omega$.

The structure of this paper is arranged as follows. In Section 2, we estimate the a priori bounds of positive solutions of (7). In Section 3, the local and global stabilities of nonnegative constant steady states of (7) are discussed. In Section 4, we give a priori estimate for the positive solutions of (8) by using maximum principle and Harnack inequality. In Section 5, we give a nonexistence result of nonconstant solutions of (8). In Section 6, we consider the existence of nonconstant positive solutions of (8). Finally, to support our theoretical predictions, some numerical simulations are given.

\section{Basic Dynamics and a Priori Bound}

Theorem 1. For system (7), one has the following.

(a) If $u_{0}(x) \geq 0, v_{0}(x) \geq 0$, then system (7) has a unique solution $(u(t, x), v(t, x))$ such that $u(t, x)>0, v(t, x)>$ 0 for $t \in(0,+\infty)$ and $x \in \bar{\Omega}$.

(b) Any solution $(u, v)$ of (7) satisfies

$$
\begin{aligned}
\limsup _{t \rightarrow+\infty} u(t, x) & \leq K, \\
\int_{\Omega} v(x, t) d x & \leq \frac{\beta}{\rho}\left(1+\frac{r_{1}}{r_{2}}\right) K|\Omega| .
\end{aligned}
$$

Proof. (a) Define

$$
\begin{aligned}
& f(u, v)=r_{1} u\left(1-\frac{u}{K}\right)-\rho u^{\alpha} v, \\
& g(u, v)=\beta u^{\alpha} v-r_{2} v .
\end{aligned}
$$

Then $f_{v}=-\rho u^{\alpha} \leq 0$ and $g_{u}=\beta \alpha u^{\alpha-1} v \geq 0$ in $\overline{\mathbb{R}_{+}^{2}}=$ $\{u \geq 0, v \geq 0\}$. Hence, (7) is a mixed qusi-monotone system. Consider following system:

$$
\begin{aligned}
\frac{d u}{d t} & =r_{1} u\left(1-\frac{u}{K}\right)-\rho u^{\alpha} v, \\
\frac{d v}{d t} & =\beta u^{\alpha} v-r_{2} v, \\
u(0) & =u_{0}, \\
v(0) & =v_{0} .
\end{aligned}
$$

Assume $u\left(t ; u_{0}, v_{0}\right), v\left(t ; u_{0}, v_{0}\right)$ are the unique solution to system (11). Let

$$
\begin{aligned}
& \max _{\bar{\Omega}} u_{0}(x)=\phi_{M}, \\
& \max _{\bar{\Omega}} v_{0}(x)=\psi_{M} .
\end{aligned}
$$

Obviously, $(\underline{u}(t, x), \underline{v}(t, x))=(0,0)$ and $(\bar{u}(t), \bar{v}(t))=(u(t ;$ $\left.\left.\phi_{M}, \psi_{M}\right), v\left(t ; \phi_{M}, \psi_{M}\right)\right)$ are a pair of lower-solution and upper-solution to system (7). Therefore, according to the Theorem 8.3.3 in [22] or Theorem 5.3.2 in [23], system (7) 
has a unique globally defined solution $(u(x, t), v(x, t))$ which satisfies

$$
\begin{aligned}
& 0 \leq u(x, t) \leq u\left(t ; \phi_{M}, \psi_{M}\right), \\
& 0 \leq v(x, t) \leq v\left(t ; \phi_{M}, \psi_{M}\right) .
\end{aligned}
$$

The strong maximum principle implies that $u(x, t), v(x, t)>$ 0 when $t>0$ for all $x \in \bar{\Omega}$.

(b) By the first equation of (7), we easily obtain the fact that $r_{1} u(1-u / K)-\rho u^{\alpha} v \leq r_{1} u(1-u / K)$ in $[0,+\infty) \times \Omega$; the first result follows easily from the simple comparison argument for parabolic problems, and thus there exists $T \in(0,+\infty)$ such that $u(t, x) \leq K+\epsilon$ in $[T,+\infty) \times \Omega$ for an arbitrary constant $\epsilon>0$.

For the estimate of $v(x, t)$, let $U(t)=\int_{\Omega} u(x, t) d x, V(t)=$ $\int_{\Omega} v(x, t) d x$; then

$$
\begin{aligned}
\frac{d U}{d t} & =\int_{\Omega} u_{t} d x \\
& =d_{1} \int_{\Omega} \Delta u d x+\int_{\Omega}\left[r_{1} u\left(u-\frac{u}{K}\right)-\rho u^{\alpha} v\right] d x \\
& =\int_{\Omega}\left[r_{1} u\left(u-\frac{u}{K}\right)-\rho u^{\alpha} v\right] d x, \\
\frac{d V}{d t} & =\int_{\Omega} v_{t} d x=d_{2} \int_{\Omega} \Delta v d x+\int_{\Omega}\left(\beta u^{\alpha} v-r_{2} v\right) d x \\
& =\int_{\Omega}\left(\beta u^{\alpha} v-r_{2} v\right) d x .
\end{aligned}
$$

Multiplying (14) by $\beta / \rho$ and adding it to (15), we have

$$
\begin{aligned}
\left(\frac{\beta}{\rho} U+V\right)_{t}= & -r_{2} V+\frac{\beta r_{1}}{\rho} \int_{\Omega} u\left(u-\frac{u}{K}\right) d x \\
\leq & -r_{2}\left(\frac{\beta}{\rho} U+V\right) \\
& +\left(\frac{\beta r_{2}}{\rho}+\frac{\beta r_{1}}{\rho}\right) \rho(K+\epsilon)|\Omega| .
\end{aligned}
$$

Integration of the inequality leads to

$$
\begin{aligned}
\int_{\Omega} v(x, t) d x & =V(t)<\frac{\beta}{\rho} U(t)+V(t) \\
& \leq \frac{\beta}{\rho}\left(1+\frac{r_{1}}{r_{2}}\right)(K+\epsilon)|\Omega| .
\end{aligned}
$$

\section{Stability of the Nonnegative Constant Steady States of (7)}

In this section, we will analyze the stability of nonnegative constant steady states of (7). By the direct computation, we see that the possible nonnegative constant steady states of (7) are

$$
\begin{aligned}
& E_{0}=(0,0), \\
& E_{1}=(K, 0), \\
& E^{*}=\left(u^{*}, v^{*}\right),
\end{aligned}
$$

where $u^{*}=\left(r_{2} / \beta\right)^{1 / \alpha}, v^{*}=r_{1} u^{*(1-\alpha)}\left(1-u^{*} / K\right) / \rho$. Obviously, the positive constant steady state $E^{*}$ exists if $r_{2}<\beta K^{\alpha}$ holds.

Notation 1. Let $0=\mu_{0}<\mu_{1}<\mu_{2}<\cdots<\mu_{n}<$ $\cdots \rightarrow \infty$ be the eigenvalues of $-\Delta$ on $\Omega$ under homogeneous Neumann boundary condition. We define the following space decomposition:

(i) $S\left(\mu_{n}\right)$ is the space of eigenfunctions corresponding to $\mu_{i}$ for $n=0,1,2, \ldots$

(ii) $X_{i j}:=\left\{\mathbf{c} \cdot \phi_{i j}: \mathbf{c} \in \mathbb{R}^{2}\right\}$, where $\left\{\phi_{i j}\right\}$ are orthonormal basis of $S\left(\mu_{n}\right)$ for $j=1,2, \ldots, \operatorname{dim}\left[S\left(\mu_{n}\right)\right]$.

(iii) $\mathbf{X}:=\left\{\mathbf{u}=(u, v) \in\left[C^{1}(\bar{\Omega})\right]^{2}: \partial u / \partial \mathbf{n}=\partial v / \partial \mathbf{n}=0\right\}$, and so $\mathbf{X}=\oplus_{i=1}^{\infty} \mathbf{X}_{i}$, where $\mathbf{X}_{i}=\oplus_{j=1}^{\operatorname{dim}\left[S\left(\mu_{j}\right)\right]} \mathbf{X}_{i j}$.

Let $\widetilde{E}$ be a nonnegative constant steady state of (7); then the linearization of (7) at a constant solution $\widetilde{E}$ can be expressed by

$$
\mathbf{u}_{t}=(D \Delta+J) \mathbf{u},
$$

where $D=\operatorname{diag}\left(d_{1}, d_{2}\right), \mathbf{u}=(u(x, t), v(x, t))^{T}$, and

$$
J=\left(\begin{array}{cc}
r_{1}-\frac{2 r_{1} u^{*}}{K}-\rho \alpha u^{*(\alpha-1)} v^{*} & -\rho u^{* \alpha} \\
-\beta v^{*} u^{*(\alpha-1)} & \beta u^{* \alpha}-r_{2}
\end{array}\right) .
$$

In view of Notation 1 , we can induce the eigenvalues of system (19) confined on the subspace $\mathbf{X}_{i}$. If $\lambda$ is an eigenvalue of (19) on $\mathbf{X}_{i}$, it must be an eigenvalue of the matrix $-\mu_{n} D+J$ for each $n \geq 0$. It is easy to see that $\lambda$ satisfies the characteristic equation

$$
\begin{aligned}
\lambda^{2}+ & \left(d_{1} \mu_{n}+d_{2} \mu_{n}-r_{1}+\frac{2 r_{1} u^{*}}{K}+\rho \alpha u^{*(\alpha-1)} v^{*}\right) \lambda \\
& +d_{2} \mu_{n}\left(d_{1} \mu_{n}-r_{1}+\frac{2 r_{1} u^{*}}{K}+\rho \alpha u^{*(\alpha-1)} v^{*}\right) \\
& +\rho \beta u^{*(2 \alpha-1)} v^{*}=0 .
\end{aligned}
$$

Theorem 2. (i) The trivial equilibrium $E_{0}=(0,0)$ is unstable. (ii) If $\beta K^{\alpha}<r_{2}$, then $E_{1}=(K, 0)$ is globally asymptotically stable.

(iii) If $1-\alpha-((2-\alpha) / K) u^{*}<0$, then $E^{*}$ is locally asymptotically stable.

Proof. (i) For $E_{0}=(0,0)$, the eigenvalues are

$$
\begin{aligned}
& \lambda_{1 n}=r_{1}-d_{1} \mu_{n}, \\
& \lambda_{2 n}=-d_{2} \mu_{n} .
\end{aligned}
$$

Obviously, $E_{0}$ is unstable. 
(ii) For $E_{1}=(K, 0)$, the eigenvalues are

$$
\begin{aligned}
& \lambda_{1 n}=-r_{1}-d_{1} \mu_{n}, \\
& \lambda_{2 n}=\beta K^{\alpha}-r_{2}-d_{2} \mu_{n} .
\end{aligned}
$$

If $\beta K^{\alpha}<r_{2}$, then $\lambda_{1 n}$ and $\lambda_{2 n}$ are all negative. Therefore $E_{1}$ is locally asymptotically stable. Indeed, $E_{1}$ is globally asymptotically stable.

On account of Theorem 1, we have lim $\sup _{t \rightarrow+\infty} \max _{\bar{\Omega}} u(\cdot$, $t) \leq K$, and thus there exists $T_{1} \in(0,+\infty)$ such that, for an arbitrary constant $0<\epsilon<\left(r_{2} / \beta\right)^{1 / \alpha}-K$,

$$
u(\cdot, t) \leq K+\epsilon, \quad t \geq T_{1} .
$$

It follows from the second equation of (7) that

$$
v_{t}-d_{2} \Delta v \leq v\left(\beta(K+\epsilon)^{\alpha}-r_{2}\right), \quad t \geq T_{1} .
$$

Therefore, $\lim \sup _{t \rightarrow+\infty} \max _{\bar{\Omega}} v(\cdot, t) \leq 0$, and there exists $T_{2}>$ $T_{1}$ such that

$$
v(\cdot, t) \leq \epsilon, \quad t \geq T_{2} .
$$

It follows from the first equation of (7) that

$$
u_{t}-d_{1} \Delta u \geq r_{1} u\left(1-\frac{u}{K}\right)-\rho u^{\alpha} \epsilon, \quad t>T_{2}, x \in \Omega .
$$

On account of and the arbitrariness of $\epsilon>0$, we have $\liminf _{t \rightarrow+\infty} \min _{\bar{\Omega}} u(\cdot, t) \geq K$. This combined with $\lim \sup _{t \rightarrow+\infty} \max _{\bar{\Omega}} u(\cdot, t) \leq K$ allows us to derive

$$
\lim _{t \rightarrow+\infty} \max _{\bar{\Omega}}|u(\cdot, t)-K|=0 .
$$

Hence, $E_{1}$ is globally asymptotically stable when $r_{2}>K^{\alpha} \beta$.

(iii) When $E^{*}=\left(u^{*}, v^{*}\right)$ exists, the corresponding characteristic equation is as follows:

$$
\begin{aligned}
\lambda^{2}+ & \left(d_{1} \mu_{n}+d_{2} \mu_{n}-r_{1}\left(1-\alpha-\frac{2-\alpha}{K} u^{*}\right)\right) \lambda \\
& +d_{2} \mu_{n}\left(d_{1} \mu_{n}-r_{1}\left(1-\alpha-\frac{2-\alpha}{K} u^{*}\right)\right) \\
& +\rho \beta u^{*(2 \alpha-1)} v^{*}=0 .
\end{aligned}
$$

Obviously, we have

$$
\begin{aligned}
\lambda_{1 n}+\lambda_{2 n}= & r_{1}\left(1-\alpha-\frac{2-\alpha}{K} u^{*}\right)-d_{1} \mu_{n}-d_{2} \mu_{n} \\
\lambda_{1 n} \lambda_{2 n}= & d_{2} \mu_{n}\left(d_{1} \mu_{n}-r_{1}\left(1-\alpha-\frac{2-\alpha}{K} u^{*}\right)\right) \\
& +\rho \beta u^{*(2 \alpha-1)} v^{*}
\end{aligned}
$$

If $1-\alpha-((2-\alpha) / K) u^{*}<0$, then $\lambda_{1 n}+\lambda_{2 n}<0$ and $\lambda_{1 n} \lambda_{2 n}>$ 0 . Hence, all the roots of (29) have negative real part which means that $E^{*}$ is locally asymptotically stable when $1-\alpha-$ $((2-\alpha) / K) u^{*}<0$.

\section{The Prior Estimate}

In this section, we will give some a priori estimates of positive solutions to (8). Firstly, we give two known lemmas.

Lemma 3 (Harnack inequality (cf. [24])). Let $\omega \in C^{2}(\Omega) \cap$ $C^{1}(\bar{\Omega})$ be a positive classical solution to

$$
\begin{aligned}
\Delta \omega(x)+c(x) \omega(x) & =0 \quad \text { in } \Omega, \\
\frac{\partial \omega}{\partial n} & =0 \quad \text { on } \partial \Omega .
\end{aligned}
$$

Then there exists a positive constant $C$ such that

$$
\max _{\bar{\Omega}} \omega \geq C \min _{\bar{\Omega}} \omega .
$$

Lemma 4 (maximum principle (cf. [25])). Suppose that $g \in$ $C(\bar{\Omega} \times \mathbb{R})$.

(i) Assume that $\omega \in C^{2}(\Omega) \cap C^{1}(\bar{\Omega})$ satisfies

$$
\begin{aligned}
\Delta \omega(x)+c(x) \omega(x) & \geq 0 \quad \text { in } \Omega, \\
\frac{\partial \omega}{\partial n} \leq 0 & \text { on } \partial \Omega .
\end{aligned}
$$

If $\omega\left(x_{0}\right)=\max _{\bar{\Omega}} \omega$, then $g\left(x_{0}, \omega\left(x_{0}\right)\right) \geq 0$.

(ii) Assume that $\omega \in C^{2}(\Omega) \cap C^{1}(\bar{\Omega})$ satisfies

$$
\begin{aligned}
\Delta \omega(x)+c(x) \omega(x) \leq 0 & \text { in } \Omega, \\
\frac{\partial \omega}{\partial n} \geq 0 & \text { on } \partial \Omega .
\end{aligned}
$$

If $\omega\left(x_{0}\right)=\min _{\bar{\Omega}} \omega$, then $g\left(x_{0}, \omega\left(x_{0}\right)\right) \leq 0$.

Lemma 5. For any positive solution $(u, v)$ of system (8),

$$
\begin{aligned}
& 0<u(x)<K, \\
& 0<v(x)<\frac{\beta}{\rho}\left(\frac{d_{1}}{d_{2}}+\frac{r_{1}}{r_{2}}\right) K
\end{aligned}
$$

for any $x \in \bar{\Omega}$.

Proof. Form Lemma $4, u(x) \leq K$ and from the strong maximum principle $u(x)<K$ for all $x \in \bar{\Omega}$. Multiplying the first equation of (8) by $\beta / \rho$ and adding it to the second equation, we have

$$
\begin{aligned}
-\left(\frac{\beta}{\rho} d_{1} \Delta u+d_{2} \Delta v\right) & =\frac{\beta}{\rho} r_{1} u\left(1-\frac{u}{K}\right)-r_{2} v \\
\leq & \frac{\beta}{\rho} r_{1} K+\frac{d_{1} r_{2} \beta}{\rho d_{2}} K \\
& -\frac{r_{2}}{d_{2}}\left(\frac{\beta}{\rho} d_{1} u+d_{2} v\right) .
\end{aligned}
$$

Then the maximum principle implies that

$$
\frac{\beta}{\rho} d_{1} u+d_{2} v \leq \frac{\beta}{\rho}\left(\frac{d_{2} r_{1}}{r_{2}}+d_{1}\right) K .
$$

Hence, $v(x)<(\beta / \rho)\left(d_{1} / d_{2}+r_{1} / r_{2}\right) K$. 
In the following, we estimate the positive lower bound of positive solution of (8).

Theorem 6. Let $\Omega$ be a bounded smooth domain in $R^{n}$. There exist two positive constants $\underline{C}<\bar{C}$ depending possibly on $d_{1}$, $d_{2}, K, \beta, \alpha, \rho$, and $\Omega$, such that such that any positive solution $(u(x), v(x))$ of system (8) satisfies

$$
\begin{aligned}
& \underline{C} \leq u(x) \leq \bar{C}, \\
& \underline{C} \leq v(x) \leq \bar{C}
\end{aligned}
$$

$$
\text { for any } x \in \bar{\Omega} \text {. }
$$

Proof. From Lemma 5, we obtain

$$
u(x), v(x) \leq \bar{C}:=\max \left\{K, \frac{\beta}{\rho}\left(\frac{d_{1}}{d_{2}}+\frac{r_{1}}{r_{2}}\right) K\right\},
$$

where $\bar{C}$ depends on $d_{1}, d_{2}, K, \beta, \alpha$, and $\rho$.

From Lemma 3 , we obtain the fact that there exists a positive constant $C_{2}$ such that

$$
\begin{aligned}
& \sup _{\bar{\Omega}} u(x) \leq C_{2} \inf _{\bar{\Omega}} u(x), \\
& \sup _{\bar{\Omega}} v(x) \leq C_{2} \inf _{\bar{\Omega}} v(x) .
\end{aligned}
$$

On the contrary, suppose the result is false. Then there exists a sequence $\left\{\left(u_{n}, v_{n}\right)\right\}$ of positive solutions to system (8) such that

$$
\begin{aligned}
\sup _{\bar{\Omega}} u_{n} \longrightarrow 0 & \\
\operatorname{or} \sup _{\bar{\Omega}} v_{n} \longrightarrow & 0 \\
& \text { as } n \longrightarrow+\infty .
\end{aligned}
$$

By the regularity theory for elliptic equations, there exists a subsequence of $\left\{\left(u_{n}, v_{n}\right)\right\}$, which will be denoted again by $\left\{\left(u_{n}, v_{n}\right)\right\}$, such that $\left\{\left(u_{n}, v_{n}\right)\right\} \rightarrow\left(u_{0}, v_{0}\right)$ in $C^{2}(\bar{\Omega})$ as $n \rightarrow$ $+\infty$. Observe that $u_{0} \leq K$ and, from (41), either $u_{0} \equiv 0$ or $v_{0} \equiv 0$. Therefore, we have the following two cases:

(i) $u_{0} \equiv 0, v_{0} \neq \equiv 0$; or $u_{0} \equiv 0, v_{0} \equiv 0$.

(ii) $u_{0} \not \equiv 0, v_{0} \equiv 0$.

Since $\left\{\left(u_{n}, v_{n}\right)\right\}$ is a positive solution of $(8)$, one can obtain the following integral equation by integrating (8) for $u_{n}$ and $v_{n}$ over $\Omega$ :

$$
\begin{array}{r}
\int_{\Omega}\left(r_{1} u_{n}\left(1-\frac{u_{n}}{K}\right)-\rho u_{n}^{\alpha} v_{n}\right) d x=0, \\
\int_{\Omega}\left(\beta u_{n}^{\alpha} v_{n}-r_{2} v_{n}\right) d x=0 .
\end{array}
$$

(i) In this case, $u_{0} \equiv 0$; then

$$
\beta u_{n}^{\alpha}-r_{2} \longrightarrow-r_{2}<0
$$

uniformly as $n \rightarrow \infty$ and $v_{n}>0$; then for sufficiently large $n$, we have

$$
\int_{\Omega}\left(\beta u_{n}^{\alpha} v_{n}-r_{2} v_{n}\right) d x<0,
$$

which is a contradiction. (ii) If $u_{0} \not \equiv 0, v_{0} \equiv 0$, then this implies that $u_{0}$ satisfies (8). So $u_{0} \equiv K$ for large $n$. Thus

$$
\beta u_{n}^{\alpha}-r_{2} \longrightarrow \beta K^{\alpha}-r_{2}>0
$$

for large $n$ since $\beta K^{\alpha}<r_{2}$, which derives a contradiction again to the second integral equation of (42). This completes the proof.

\section{Nonexistence of Nonconstant Positive Steady States}

In this section, we can show the nonexistence of nonconstant positive solutions to system (8) when the diffusion coefficients $d_{1}$ and $d_{2}$ are large.

Theorem 7. There exists a positive constant $d^{*}$ such that elliptic problem (8) has no nonconstant positive solution if $\min \left\{d_{1}, d_{2}\right\}>d^{*}$.

Proof. Suppose that $(u(x), v(x))$ is a nonconstant positive solution of system (8). Denote $\bar{u}=|\Omega|^{-1} \int_{\Omega} u(x) d x \geq 0$, $\bar{v}=|\Omega|^{-1} \int_{\Omega} u(x) d x \geq 0$. Then

$$
\begin{aligned}
& \int_{\Omega}(u-\bar{u}) d x=0 \\
& \int_{\Omega}(v-\bar{v}) d x=0 .
\end{aligned}
$$

Define

$$
h(u)=\frac{\bar{u}-u}{\bar{u}^{\alpha}-u^{\alpha}}
$$

for $\bar{u} \neq u$. Indeed, we can prove that $h(u)>0$ and $h^{\prime}(u)>0$. In fact, notice

$$
h^{\prime}(u)=\frac{(1-\alpha) u^{\alpha}+\alpha \bar{u} u^{(\alpha-1)}-\bar{u}^{\alpha}}{\left(\bar{u}^{\alpha}-u^{\alpha}\right)^{2}} .
$$

Let

$$
h_{1}(u)=(1-\alpha) u^{\alpha}+\alpha \bar{u} u^{(\alpha-1)}-\bar{u}^{\alpha}
$$

and we have

$$
h_{1}^{\prime}(u)=\alpha(1-\alpha) u^{(\alpha-2)}(u-\bar{u}),
$$

which implies that $h_{1}(u)>\min h_{1}(u)=h_{1}(\bar{u})=0$ for $u \neq \bar{u}$. Therefore, we obtain the fact that $h(u)>0$.

Furthermore, multiplying the first equation of (8) by $\beta / \rho$, adding it to the second equation, and integrating over $\Omega$, we get

$$
\begin{aligned}
& -\int_{\Omega}\left(\frac{\beta}{\rho} d_{1} \Delta u+d_{2} \Delta v\right) d x \\
& \quad=\int_{\Omega}\left(\frac{\beta}{\rho} r_{1} u\left(1-\frac{u}{K}\right)-r_{2} v\right) d x,
\end{aligned}
$$


and then the Neumann boundary conditions lead to

$$
r_{2} \int_{\Omega} v d x=\int_{\Omega} \frac{\beta}{\rho} r_{1} u\left(1-\frac{u}{K}\right) d x \leq \frac{K \beta r_{1}}{4 \rho}|\Omega| .
$$

Thus

$$
\bar{v}=\frac{1}{\Omega} \int_{\Omega} v d x \leq \frac{K \beta r_{1}}{4 r_{2} \rho} .
$$

Multiplying the first equation in (8) by $u-\bar{u}$, we have

$$
\begin{aligned}
& d_{1} \int_{\Omega}|\nabla(u-\bar{u})|^{2} d x=\int_{\Omega}(u-\bar{u})\left(r_{1} u\left(1-\frac{u}{K}\right)\right. \\
& \left.\quad-\rho u^{\alpha} v\right) d x=\int_{\Omega}(u-\bar{u})\left(r_{1}(u-\bar{u})\right. \\
& \left.\quad-\frac{r_{1}}{K}\left(u^{2}-\bar{u}^{2}\right)-\rho u^{\alpha} v+\rho \bar{u}^{\alpha} \bar{v}\right) d x \\
& \quad \leq 3 r_{1} \int_{\Omega}(u-\bar{u})^{2} d x \\
& +\rho K^{\alpha} \int_{\Omega}|(u-\bar{u})(v-\bar{v})| d x+\rho \int_{\Omega} \frac{\bar{v}}{h(u)}(u \\
& -\bar{u})^{2} d x \leq\left(3 r_{1}+\frac{\rho K^{\alpha}}{2}+\frac{K \beta r_{1}}{4 r_{2} h(0)}\right) \int_{\Omega}(u \\
& -\bar{u})^{2} d x+\frac{\rho K^{\alpha}}{2} \int_{\Omega}(v-\bar{v})^{2} d x .
\end{aligned}
$$

Multiplying the second equation in (8) by $v-\bar{v}$, we have

$$
\begin{gathered}
d_{2} \int_{\Omega}|\nabla(v-\bar{v})|^{2} d x=\int_{\Omega}(v-\bar{v})\left(\beta u^{\alpha} v-r_{2} v\right) d x \\
=\int_{\Omega}(v-\bar{v})\left(-r_{2}(v-\bar{v})+\beta u^{\alpha} v-\beta \bar{u}^{\alpha} \bar{v}\right) d x \\
\leq\left(r_{2}+\beta K^{\alpha}+\frac{K \beta^{2} r_{1}}{8 r_{2} \rho h(0)}\right) \int_{\Omega}(v-\bar{v})^{2} d x \\
\quad+\frac{K \beta^{2} r_{1}}{8 r_{2} \rho h(0)} \int_{\Omega}(u-\bar{u})^{2} d x .
\end{gathered}
$$

From (54) and (55) and the Poincaré inequality, we obtain

$$
\begin{aligned}
& d_{1} \int_{\Omega}|\nabla(u-\bar{u})|^{2} d x+d_{2} \int_{\Omega}|\nabla(v-\bar{v})|^{2} d x \\
& \leq A \int_{\Omega}(u-\bar{u})^{2} d x+B \int_{\Omega}(v-\bar{v})^{2} d x \\
& \leq \frac{1}{\mu_{1}}\left(A \int_{\Omega}|\nabla(u-\bar{u})|^{2} d x+B \int_{\Omega}|\nabla(v-\bar{v})|^{2} d x\right),
\end{aligned}
$$

where

$$
\begin{aligned}
& A=3 r_{1}+\frac{\rho K^{\alpha}}{2}+\frac{K \beta r_{1}}{4 r_{2} h(0)}+\frac{K \beta^{2} r_{1}}{8 r_{2} \rho h(0)}, \\
& B=r_{2}+\beta K^{\alpha}+\frac{K \beta^{2} r_{1}}{8 r_{2} \rho h(0)}+\frac{\rho K^{\alpha}}{2} .
\end{aligned}
$$

Hence, if

$$
\min \left\{d_{1}, d_{2}\right\}>d^{*}:=\frac{1}{\mu_{1}} \max \{A, B\},
$$

then

$$
\nabla(u-\bar{u})=\nabla(v-\bar{v})=0,
$$

and $(u, v)$ must be a constant solution.

\section{Existence of Nonconstant Positive Steady States}

In this subsection, we discuss the existence of nonconstant positive solutions to system (8) when the diffusion coefficients $d_{1}$ and $d_{2}$ vary while the parameters $r_{1}, K, \alpha, \rho, \beta$, and $r_{2}$ are fixed by using the Leray-Schauder degree theory. Throughout this section, we assume that the positive constant steady state $E^{*}=\left(u^{*}, v^{*}\right)$ exists.

For simplicity, denote $\mathbf{u}=(u, v)$ and

$$
\begin{aligned}
& a_{1}=r_{1}-\frac{2 r_{1} u^{*}}{K}-\rho \alpha\left(u^{*}\right)^{(\alpha-1)} v^{*}, \\
& a_{2}=\rho\left(u^{*}\right)^{\alpha}, \\
& a_{3}=\beta v^{*}\left(u^{*}\right)^{(\alpha-1)}, \\
& \Phi(\mathbf{u})=\left(\begin{array}{c}
r_{1} u\left(1-\frac{u}{K}\right)-\rho u^{\alpha} v \\
\beta u^{\alpha} v-r_{2} v
\end{array}\right), \\
& \Phi_{\mathbf{u}}\left(E^{*}\right)=\left(\begin{array}{cc}
a_{1} & -a_{2} \\
a_{3} & 0
\end{array}\right), \\
& D=\operatorname{diag}\left\{d_{1}, d_{2}\right\}, \\
& X=\left\{(u, v) \in\left[C^{1}(\bar{\Omega})\right]^{2}: \frac{\partial u}{\partial n}=\frac{\partial v}{\partial n}=0 \text { on } \partial \Omega\right\}, \\
& X^{+}=\{(u, v): u, v \geq 0,(u, v) \in X\}, \\
& \Lambda=\left\{(u, v) \in X: C^{-1}<u, v<C \text { on } \bar{\Omega}\right\} .
\end{aligned}
$$

Thus, (8) can be written as

$$
-D \Delta \mathbf{u}=\Phi(\mathbf{u}) \quad \text { in } \frac{\partial \mathbf{u}}{\partial n}=0 \text { on } \Omega,
$$

and, obviously, $\mathbf{u}$ is a positive solution of (61) if and only if

$$
\mathscr{F}(\mathbf{u}):=\mathbf{u}-(I-\Delta)^{-1}\left(D^{-1} \Phi(\mathbf{u})+\mathbf{u}\right) \quad \text { in } X^{+},
$$

where $(I-\Delta)^{-1}$ is the inverse of $I-\Delta$ with the homogeneous Neumann boundary condition. As $\mathscr{F}(\cdot)$ is a compact perturbation of the identity operator, the Leray-Schauder degree $\operatorname{deg}(\mathscr{F}(\cdot), \Lambda, 0)$ is well-defined from Theorem 6. By direct computation, we have

$$
\mathscr{F}_{\mathbf{u}}\left(E^{*}\right)=I-(I-\Delta)^{-1}\left(D^{-1} \Phi_{\mathbf{u}}\left(E^{*}\right)+I\right) .
$$


If $\mathscr{F}_{\mathbf{u}}\left(E^{*}\right)$ is invertible, the index of $\mathscr{F}$ is defined as

$$
\operatorname{index}\left(\mathscr{F}(\cdot), E^{*}\right)=(-1)^{\gamma},
$$

where $\gamma$ is the number of negative eigenvalues of $\mathscr{F}_{\mathbf{u}}\left(E^{*}\right)$. Note that $\lambda$ is an eigenvalue of $\mathscr{F}_{\mathbf{u}}\left(E^{*}\right)$ on $X_{j}$ if and only if it is an eigenvalue of the matrix

$$
\begin{aligned}
B_{j} & =I-\frac{1}{1+\mu_{j}}\left[D^{-1} \Phi_{\mathbf{u}}\left(E^{*}\right)+I\right] \\
& =\frac{1}{1+\mu_{j}}\left[\mu_{j} I-D^{-1} \Phi_{\mathbf{u}}\left(E^{*}\right)\right] .
\end{aligned}
$$

Thus $\mathscr{F}_{\mathbf{u}}\left(E^{*}\right)$ is invertible if and only if, for all $j \geq 0$, the matrix $B_{j}$ is nonsingular. Writing

$$
\begin{aligned}
H\left(d_{1}, d_{2} ; \mu\right) & =\operatorname{det}\left[\mu I-D^{-1} \Phi_{\mathbf{u}}\left(E^{*}\right)\right] \\
& =\frac{1}{d_{1} d_{2}} \operatorname{det}\left[\mu D-\Phi_{\mathbf{u}}\left(E^{*}\right)\right],
\end{aligned}
$$

we have that if $H\left(d_{1}, d_{2} ; \mu\right) \neq 0$, then $H\left(d_{1}, d_{2} ; \mu\right)<0$ if and only if the number of negative eigenvalues of $\mathscr{F}_{\mathbf{u}}\left(E^{*}\right)$ in $X_{j}$ is odd. The following lemma gives the explicit formula of calculating the index.

Lemma 8. If $H\left(d_{1}, d_{2} ; \mu_{i}\right) \neq 0$ for all $i \geq 0$, then

$$
\begin{aligned}
& \operatorname{index}\left(\mathscr{F}(\cdot), E^{*}\right)=(-1)^{\gamma}, \\
& \gamma=\sum_{i \geq 0, H\left(d_{1}, d_{2} ; \mu\right)<0} m\left(\mu_{i}\right),
\end{aligned}
$$

where $m\left(\mu_{i}\right)$ is the algebraic multiplicity of $\mu_{i}$.

To facilitate our computation of $\operatorname{deg}\left(\mathscr{F}(\cdot), E^{*}\right)$, we only need consider the sign of $\operatorname{det}\left[\mu D-\Phi_{\mathbf{u}}\left(E^{*}\right)\right]$. The direct calculation gives

$$
\operatorname{det}\left[\mu D-\Phi_{\mathbf{u}}\left(E^{*}\right)\right]=d_{1} d_{2} \mu^{2}-a_{1} d_{2} \mu+a_{2} a_{3} .
$$

Obviously, nonnegative roots of (68) exist if and only if $a_{1}^{2} d_{2}-$ $4 d_{1} a_{2} a_{3}>0$ and $a_{1}>0$. Assume that $\mu^{+}$and $\mu^{-}$are the two roots of (68), we have the following conclusion.

Theorem 9. Assuming that $\beta K^{\alpha}>r_{2}$,

$$
\frac{d_{2}}{d_{1}}>\frac{4 a_{2} a_{3}}{a_{1}^{2}}, \quad a_{1}>0,
$$

and there exist $i, j \in N$, such that $0 \leq \mu_{j}<\mu^{-}<\mu_{j+1} \leq \mu_{i}<$ $\mu^{+}<\mu_{i+1}$ and $\sum_{k=j+1}^{i} m\left(\mu_{k}\right)$ is odd, then (8) has at least one nonconstant positive solution.

Proof. For $t \in[0,1]$, we define

$$
\mathscr{A}_{t}(\mathbf{u}) \triangleq(-\Delta+I)^{-1}\left(\begin{array}{c}
u+\left(\frac{1-t}{d^{*}}+\frac{t}{d_{1}}\right) f(u, v) \\
v+\left(\frac{1-t}{d^{*}}+\frac{t}{d_{2}}\right) g(u, v)
\end{array}\right),
$$

where $d^{*}$ is defined in Theorem 7 .
The positive solutions of the problem

$$
\begin{gathered}
\mathscr{A}_{t}(\mathbf{u})=\mathbf{u} \quad \text { in } \Omega, \\
\frac{\partial \mathbf{u}}{\partial n}=0 \quad \text { on } \partial \Omega
\end{gathered}
$$

are contained in $\Lambda$. Note that $\mathbf{u}$ is a positive solution of system (8) if and only if it is a positive solution of (71) with $t=1 . \mathbf{u}^{*}$ is the unique positive constant solution of (71) for any $t \in[0,1]$. According to the choice of $d^{*}$ in Theorem 7 , we have $E^{*}$ which is the only fixed point of $\mathscr{A}_{0}$.

$$
\operatorname{deg}\left(I-\mathscr{A}_{0}, \Lambda, 0\right)=\operatorname{index}\left(I-\mathscr{A}_{0}, \Lambda, E^{*}\right)=1 .
$$

Since $\mathscr{F}=I-H(\cdot, 1)$ and if $(8)$ has no other solutions except the constant one $E^{*}$, then we have

$$
\begin{aligned}
& \operatorname{deg}\left(I-\mathscr{A}_{1}, \Lambda,(0,0)\right)=\operatorname{index}\left(\mathscr{F}, E^{*}\right) \\
& =(-1)^{\sum_{k=j+1}^{i} m\left(\mu_{k}\right)}=-1 .
\end{aligned}
$$

On the other hand, by the homotopy invariance of the topological degree,

$$
\operatorname{deg}\left(I-\mathscr{A}_{0}, \Lambda, 0\right)=\operatorname{deg}\left(I-\mathscr{A}_{1}, \Lambda, 0\right),
$$

which is a contradiction. Therefore, there exists at least one nonconstant solution of (8).

\section{Numerical Simulation}

7.1. Global Stability of Equilibrium $E_{1}$. Consider system (7) with following parameters: $d_{1}=0.8, d_{2}=0.9, r_{1}=0.9, \beta=$ $0.1, r_{2}=0.2, K=2, \rho=0.1$, and $\alpha=2 / 3$. According to the discussions in Section 3, the steady state $E_{1}$ is globally asymptotically stable; see Figure 1.

7.2. Stability of Positive Steady State $E^{*}$. Consider system (7) with following parameters: $d_{1}=0.8, d_{2}=0.9, r_{1}=0.9, \beta=$ $0.3, r_{2}=0.2, K=2, \rho=0.1$, and $\alpha=2 / 3$. According to the discussions in Section 3, the positive steady state $E^{*}$ is locally asymptotically stable; see Figure 2 .

\section{Conclusions}

In this paper, we have investigated the existence/nonexistence of nonconstant positive steady states for a diffusive predatorprey system with a group defense for prey under Neumann boundary conditions. The existence results provide a theoretical support for pattern formation caused by diffusion. We also study the stability of nonnegative equilibria and obtain the fact that $E_{1}$ is globally asymptotically stable when $\beta K^{\alpha}<$ $r_{2}$. In fact, the positive steady state does not exist at this time. If $\beta K^{\alpha}>r_{2}$ and $1-\alpha-((2-\alpha) / K) u^{*}<0$, then the positive steady state $E^{*}$ is locally asymptotically stable. It is easily obtained that when $1-\alpha-((2-\alpha) / K) u^{*}=0$, characteristic equation (29) has a pair of purely imaginary roots. Therefore, system (7) occurs with Hopf bifurcation, as shown in Figure 3. 

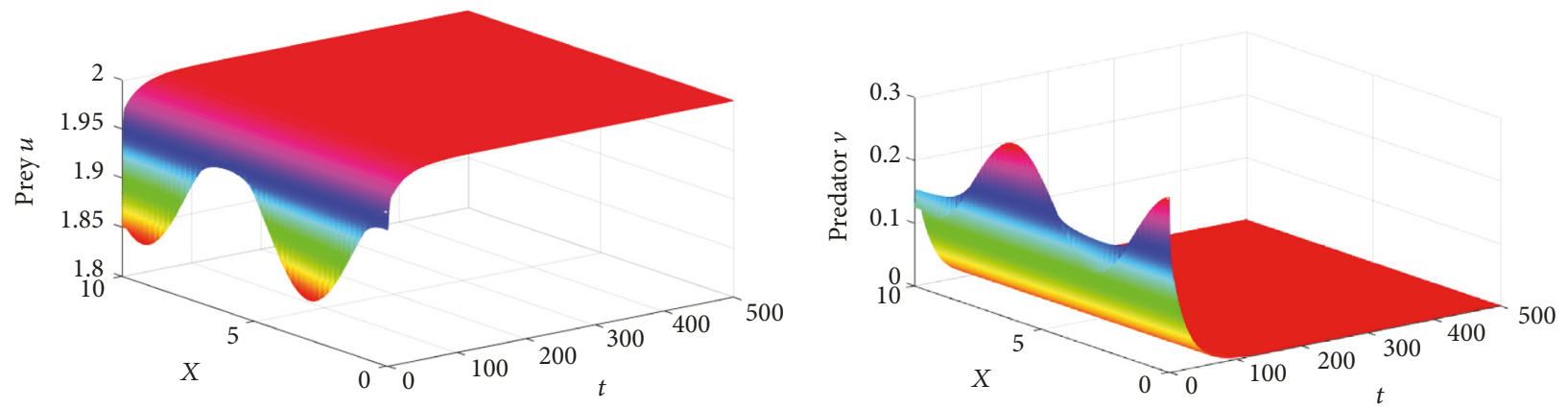

FIGURE 1: The steady state $E_{1}$ is globally asymptotically stable.
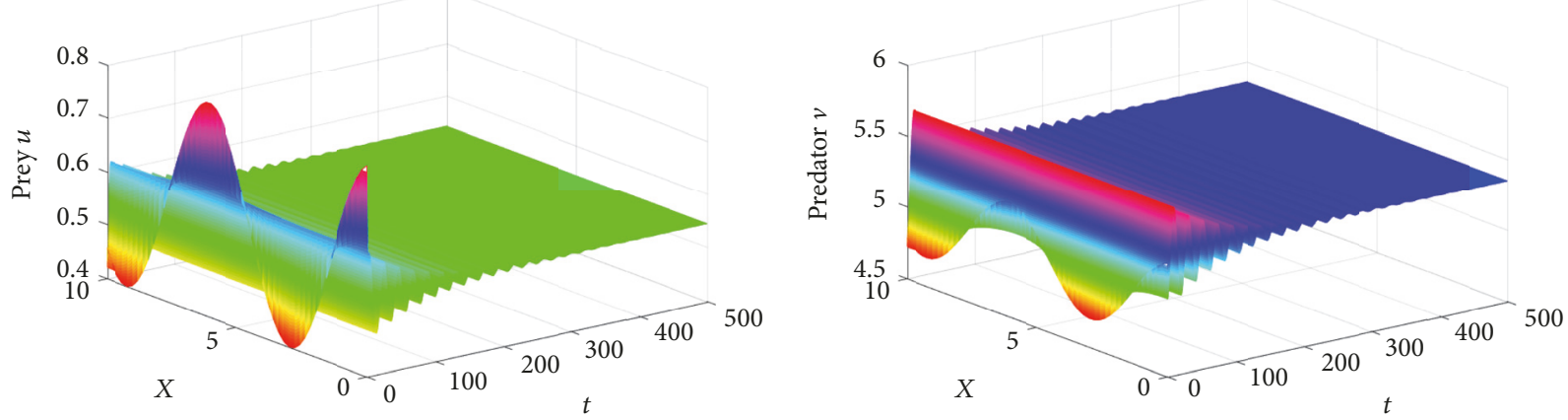

FIGURE 2: The positive steady state $E^{*}$ is locally asymptotically stable.
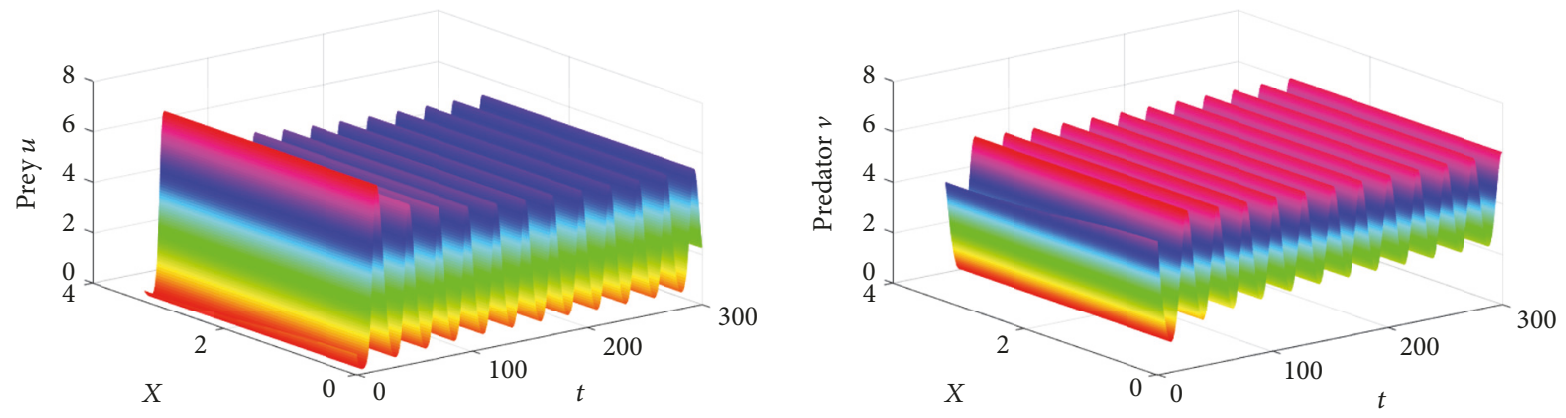

FIGURE 3: Hopf bifurcation occurs with parameters $r_{1}=0.9, \alpha=2 / 3, \beta=0.1, r_{2}=0.18, K=10 ; \rho=0.2$.

\section{Conflicts of Interest}

The authors declare that they have no conflicts of interest.

\section{Acknowledgments}

The work is sponsored by Natural Science Foundation of Jiangsu Province (CN) (BK20150420).

\section{References}

[1] L. AlfredJames, Elements of Physical Biology. Applied Mathematical Sciences, Williams and Wilkins Company, Baltimore, Maryland, 1925.
[2] V. Volterra, "Fluctuations in the abundance of a species considered mathematically," Nature, vol. 118, no. 2972, pp. 558-560, 1926.

[3] Z. Hu, Z. Teng, and L. Zhang, "Stability and bifurcation analysis of a discrete predator-prey model with nonmonotonic functional response," Nonlinear Analysis: Real World Applications, vol. 12, no. 4, pp. 2356-2377, 2011.

[4] W. Zuo and J. Wei, "Stability and Hopf bifurcation in a diffusive predatory-prey system with delay effect," Nonlinear Analysis: Real World Applications, vol. 12, no. 4, pp. 1998-2011, 2011.

[5] Y. Song and J. Wei, "Local Hopf bifurcation and global periodic solutions in a delayed predator-prey system," Journal of Mathematical Analysis and Applications, vol. 301, no. 1, pp. 1-21, 2005. 
[6] M. Baurmann, T. Gross, and U. Feudel, "Instabilities in spatially extended predator-prey systems: spatio-temporal patterns in the neighborhood of Turing-Hopf bifurcations," Journal of Theoretical Biology, vol. 245, no. 2, pp. 220-229, 2007.

[7] L. I. Xue-Peng, "Wen Sheng Yang. Global asymptotic stability of a diffusive predator-prey model with ratio-dependent functional response," Applied Mathematics Computation, vol. 250, no. 7, pp. 71-77, 2015.

[8] J.-c. Huang, "Bifurcations in a predator-prey system with Holling type-IV functional response," Journal of Central China Normal University. Natural Sciences. Huazhong Shifan Daxue Xuebao. Ziran Kexue Ban, vol. 36, no. 1, pp. 3-10, 2002.

[9] X.-P. Yan and C.-H. Zhang, "Stability and turing instability in a diffusive predator-prey system with Beddington-DeAngelis functional response," Nonlinear Analysis: Real World Applications, vol. 20, pp. 1-13, 2014.

[10] Y. Yang, S. Ruan, and D. Xiao, "Global stability of an agestructured virus dynamics model with Beddington-DeAngelis infection function," Mathematical Biosciences and Engineering, vol. 12, no. 4, pp. 859-877, 2015.

[11] V. Ajraldi, M. Pittavino, and E. Venturino, "Modeling herd behavior in population systems," Nonlinear Analysis: Real World Applications, vol. 12, no. 4, pp. 2319-2338, 2011.

[12] P. A. Braza, "Predator-prey dynamics with square root functional responses," Nonlinear Analysis: Real World Applications, vol. 13, no. 4, pp. 1837-1843, 2012.

[13] E. Venturino and S. Petrovskii, "Spatiotemporal behavior of a prey-predator system with a group defense for prey," Ecological Complexity, vol. 14, pp. 37-47, 2013.

[14] Y. Lv, Y. Pei, and R. Yuan, "Hopf bifurcation and global stability of a diffusive Gause-type predator-prey models," Computers \& Mathematics with Applications, vol. 72, no. 10, pp. 2620-2635, 2016.

[15] C. Xu, S. Yuan, and T. Zhang, "Global dynamics of a predator-prey model with defense mechanism for prey," Applied Mathematics Letters, vol. 62, pp. 42-48, 2016.

[16] S. P. Bera, A. Maiti, and G. Samanta, "Stochastic analysis of a prey-predator model with herd behaviour of prey," Lithuanian Association of Nonlinear Analysts. Nonlinear Analysis: Modelling and Control, vol. 21, no. 3, pp. 345-361, 2016.

[17] C. Q. Xu and S. L. Yuan, "Stability and Hopf bifurcation in a delayed predator-prey system with herd behavior," Abstract and Applied Analysis, vol. 2014, Article ID 568943, 8 pages, 2014.

[18] S. M. Salman, A. M. Yousef, and A. A. Elsadany, "Stability, bifurcation analysis and chaos control of a discrete predatorprey system with square root functional response," Chaos, Solitons \& Fractals, vol. 93, pp. 20-31, 2016.

[19] X. Tang and Y. Song, "Cross-diffusion induced spatiotemporal patterns in a predator-prey model with herd behavior," Nonlinear Analysis: Real World Applications, vol. 24, pp. 36-49, 2015.

[20] X. Tang and Y. Song, "Bifurcation analysis and Turing instability in a diffusive predator-prey model with herd behavior and hyperbolic mortality," Chaos, Solitons \& Fractals, vol. 81, pp. 303-314, 2015.

[21] X. Tang, Y. Song, and T. Zhang, “Turing-Hopf bifurcation analysis of a predator-prey model with herd behavior and crossdiffusion," Nonlinear Dynamics, vol. 86, no. 1, pp. 73-89, 2016.

[22] C. V. Pao, On Nonlinear Parabolic and Elliptic Equations, Plenum Press, New York, NY, USA, 1992.

[23] Q. X. Ye and Z. Y. Li, Introduction to Reaction-Diffusion Equations, Science Press, Beijing, China, 1990.
[24] C.-S. Lin, W.-M. Ni, and I. Takagi, "Large amplitude stationary solutions to a chemotaxis system," Journal of Differential Equations, vol. 72, no. 1, pp. 1-27, 1988.

[25] Y. Lou and W.-M. Ni, "Diffusion, self-diffusion and crossdiffusion," Journal of Differential Equations, vol. 131, no. 1, pp. 79-131, 1996. 


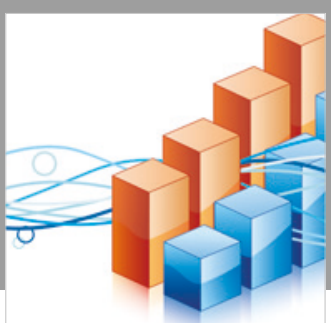

Advances in

Operations Research

\section{-n-m}
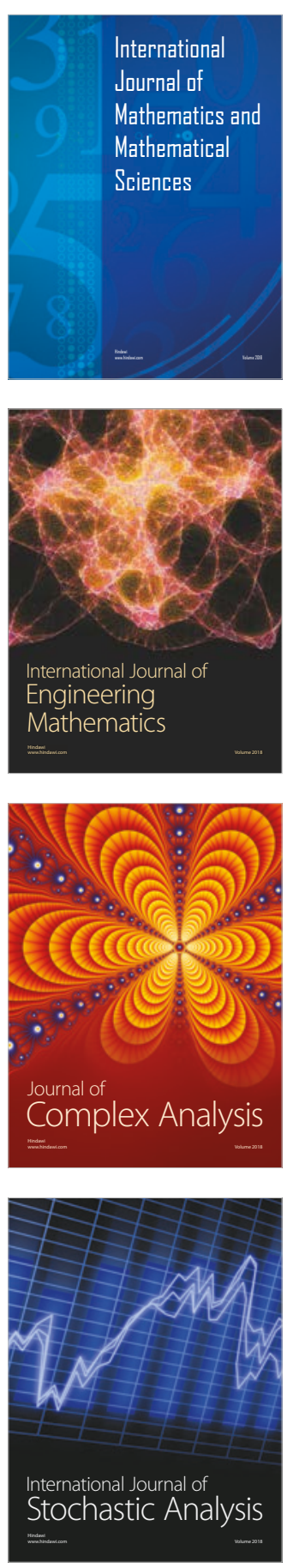
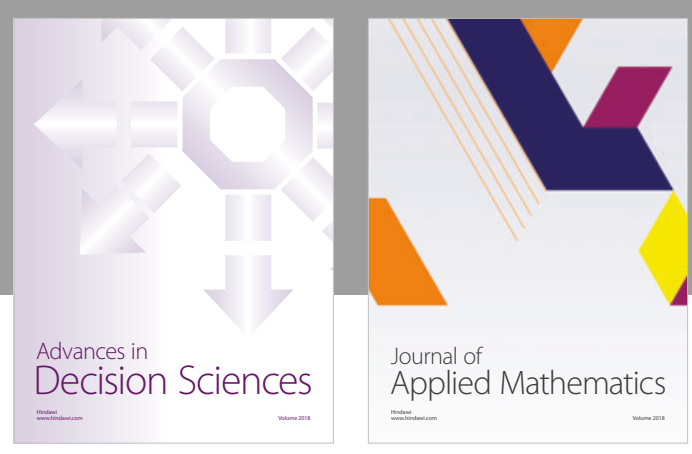

Journal of

Applied Mathematics
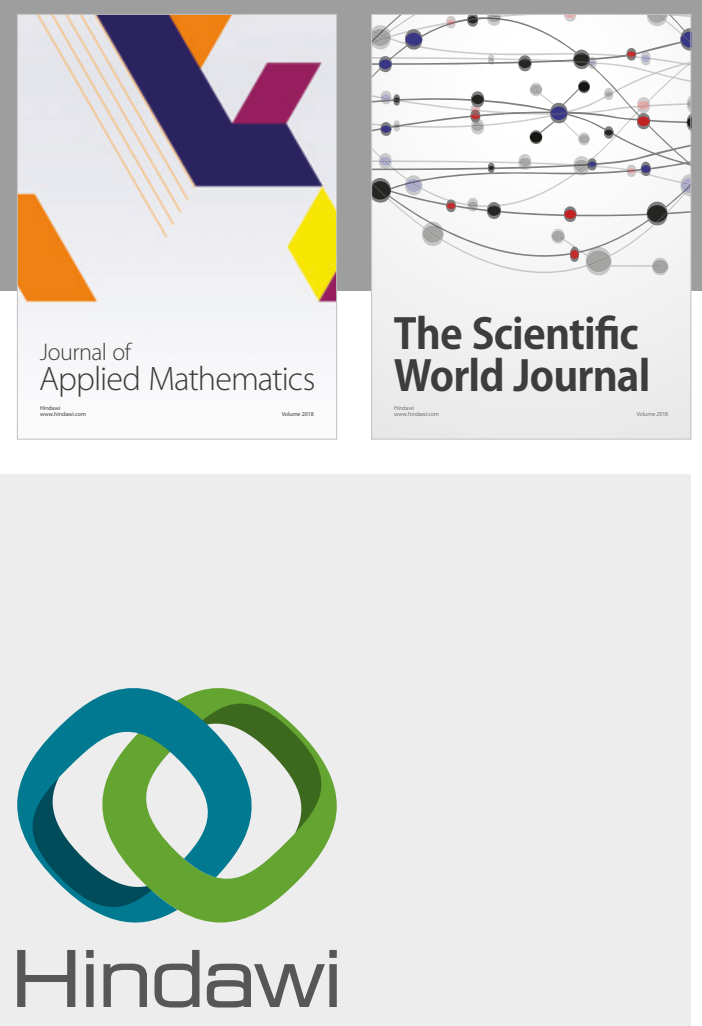

Submit your manuscripts at

www.hindawi.com

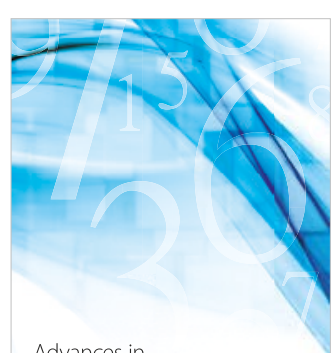

Advances in
Numerical Analysis
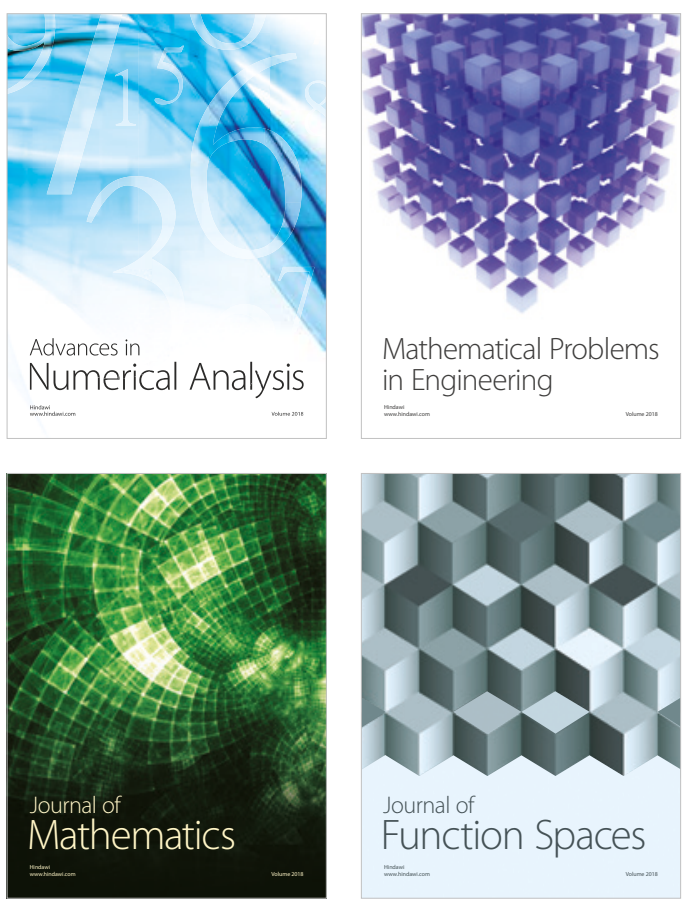

Mathematical Problems in Engineering

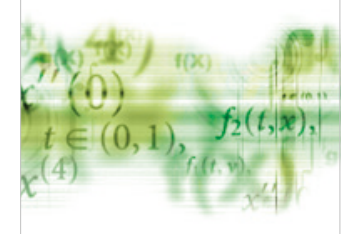

International Journal of

Differential Equations

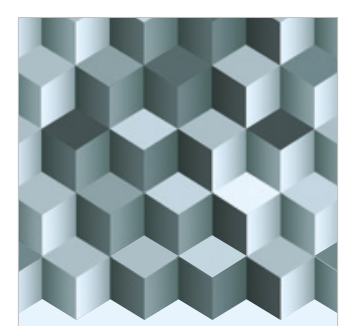

Journal of

Function Spaces

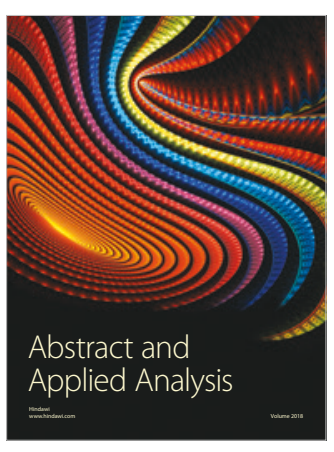

The Scientific

World Journal

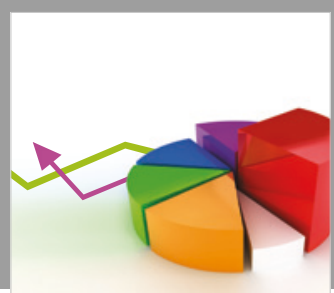

Journal of

Probability and Statistics
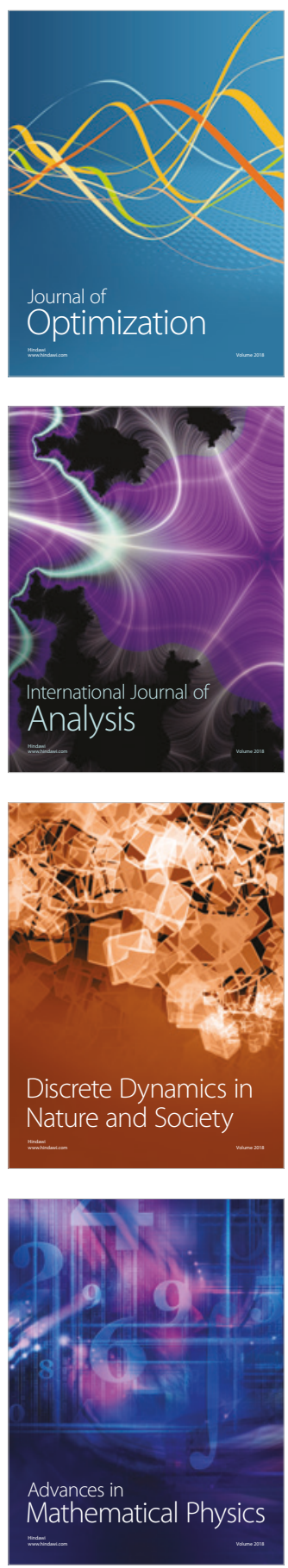